\title{
Normal insulin-dependent activation of Akt/protein kinase B, with diminished activation of phosphoinositide 3-kinase, in muscle in type 2 diabetes
}

\author{
Young-Bum Kim, ${ }^{1}$ Svetlana E. Nikoulina, ${ }^{2}$ Theodore P. Ciaraldi, ${ }^{2}$ Robert R. Henry, ${ }^{2}$ \\ and Barbara B. Kahn ${ }^{1}$ \\ ${ }^{1}$ Diabetes Unit, Department of Medicine, Beth Israel Deaconess Medical Center and Harvard Medical School, Boston, \\ Massachusetts 02215, USA \\ ${ }^{2}$ Department of Medicine, University of California-San Diego and Veterans Affairs San Diego Health Care System, San Diego, \\ California 92161, USA \\ Address correspondence to: Barbara B. Kahn, Diabetes Unit, Beth Israel Deaconess Medical Center, 330 Brookline Avenue, \\ Boston, Massachusetts 02215, USA. Phone: (617) 667-5422; Fax: (617) 667-2927; E-mail: bkahn@caregroup.harvard.edu. \\ Received for publication March 29, 1999, and accepted in revised form August 3, 1999.
}

To determine whether the serine/threonine kinase Akt (also known as protein kinase B) is activated in vivo by insulin administration in humans, and whether impaired activation of Akt could play a role in insulin resistance, we measured the activity and phosphorylation of Akt isoforms in skeletal muscle from 3 groups of subjects: lean, obese nondiabetic, and obese type 2 diabetic. Vastus lateralis biopsies were taken in the basal (overnight fast) and insulin-stimulated (euglycemic clamp) states. Insulin-stimulated glucose disposal was reduced $31 \%$ in obese subjects and $63 \%$ in diabetic subjects, compared with lean subjects. Glycogen synthase (GS) activity in the basal state was reduced $28 \%$ in obese subjects and $49 \%$ in diabetic subjects, compared with lean subjects. Insulin-stimulated GS activity was reduced $30 \%$ in diabetic subjects. Insulin treatment activated the insulin receptor substrate-1-associated (IRS-1-associated) phosphoinositide 3-kinase (PI 3-kinase) 6.1-fold in lean, 3.7-fold in obese, and 2.4-fold in diabetic subjects. Insulin also stimulated IRS-2-associated PI 3-kinase activity 2.2-fold in lean subjects, but only 1.4-fold in diabetic subjects. Basal activity of Akt1/Akt2 (Akt1/2) and Akt3 was similar in all groups. Insulin increased Akt1/2 activity 1.7- to 2.0-fold, and tended to activate Akt3, in all groups. Insulin-stimulated phosphorylation of Akt1/2 was normal in obese and diabetic subjects. In lean subjects only, insulin-stimulated Akt1/2 activity correlated with glucose disposal rate. Thus, insulin activation of Akt isoforms is normal in muscle of obese nondiabetic and obese diabetic subjects, despite decreases of approximately $50 \%$ and $39 \%$ in IRS-1- and IRS-2-associated PI 3-kinase activity, respectively, in obese diabetic subjects. It is therefore unlikely that Akt plays a major role in the resistance to insulin action on glucose disposal or GS activation that is observed in muscle of obese type 2 diabetic subjects.

J. Clin. Invest. 104:733-741 (1999).

\section{Introduction}

A fundamental mechanism for maintenance of glucose homeostasis is the rapid action of insulin to stimulate glucose uptake and metabolism in peripheral tissues. Skeletal muscle is the primary site of glucose disposal in the insulin-stimulated state (1). Resistance to the actions of insulin in skeletal muscle is a major pathogenic factor in type 2, or non-insulin-dependent, diabetes mellitus (NIDDM) (2). This resistance also contributes to the morbidity of obesity, and complicates poorly controlled type 1 (autoimmune) diabetes (3). Insulin increases glucose transport in skeletal muscle by eliciting the translocation of GLUT4, the major insulin-regulated glucose transporter, from intracellular vesicles to the plasma membrane and transverse tubules $(4,5)$. In muscle of type 2 diabetic subjects, the expression of the GLUT4 gene is normal; impaired insulin action on glucose uptake most likely results from altered trafficking or impaired function of GLUT4 (6). Because glucose transport in response to other stimuli that use different signaling pathways is normal in muscle of type 2 diabetic subjects $(7,8)$, the resistance to insulin stimulation may be due to impaired insulin signal transduction (9). Although there is growing information about the proximal steps in insulin signaling $(10,11)$, the more distal pathways involved in insulin-stimulated glucose uptake are still unclear.

Insulin signaling involves a cascade of events initiated by insulin binding to its cell-surface receptor. This is followed by receptor autophosphorylation and activation of receptor tyrosine kinases, which result in tyrosine phosphorylation of insulin receptor substrates (IRSs), including IRS-1, IRS-2, IRS-3, IRS-4, Gab1, and Shc (4, $5,12,13)$. Binding of IRSs to the regulatory subunit of phosphoinositide 3-kinase (PI 3-kinase) at Src homology 2 domains results in activation of PI 3-kinase, which is necessary for insulin action on glucose transport (14-17), glycogen synthase (GS) (18), protein synthesis (19), antilipolysis (15), and gene expression (20). PI 3kinase activation is responsible, at least in part, for insulin stimulation of GLUT4 translocation from intracellular vesicles to the plasma membrane $(15,21,22)$. Insulin-stimulated PI 3-kinase activity is decreased in lean type 2 diabetic subjects, providing evidence for a defect in insulin signaling that could contribute to 
impaired GLUT4 translocation and insulin resistance (9). However, the downstream pathways by which impaired insulin-stimulated PI 3-kinase activation results in decreased GLUT4 translocation remain unknown. A candidate molecule of recent interest is the serine/threonine kinase Akt, also known as protein kinase $\mathrm{B}$, or Rac.

Akt is a proto-oncogene with homology to protein kinases $A$ and $C(23,24)$. Insulin and other growth factors activate Akt through PI 3-kinase, although other agonists can activate Akt by a PI 3-kinase-independent pathway (25-27). Activation of Akt requires phosphorylation at threonine and serine residues (28). This phosphorylation is brought about by protein kinases such as the recently identified phosphoinositidedependent protein kinase-1 (29). Three isoforms of Akt have been cloned. In rodent muscle, Akt 1 and to a lesser extent Akt 2 are stimulated by insulin, whereas Akt3 shows minimal response (30).

Akt mediates the effects of PI 3-kinase on some cellular events, such as apoptosis (31) and protein synthesis $(32,33)$. It is also thought to mediate the phosphorylation and inactivation of glycogen synthase kinase-3 (GSK-3) by insulin (34). GSK-3 is a negative regulator of glycogen synthesis through its inhibitory effect on GS activity (35). The role of Akt in GLUT4 translocation is controversial $(32,36-38)$. Overexpression of constitutively active Akt results in increased glucose uptake in the absence of insulin in 3T3-L1 adipocytes $(22,35)$, and stimulates GS activity by inhibition of GSK-3 activity in L6 myotubes (39). However, a dominant-negative Akt in which both phosphorylation sites were mutated blocked protein synthesis, but did not impair insulin activation of glucose transport (31). In contrast, a construct that was also mutated in the kinase domain blocked insulinstimulated GLUT4 translocation (38). When muscle strips from lean diabetic subjects were incubated in vitro with physiologic concentrations of insulin, activation of Akt (detected with an antibody against the pleckstrin homology domain of Akt 1 ) was normal - although at pharmacologic levels of insulin, Akt activation was modestly reduced (40). Whether activation of Akt in vivo is impaired in insulin-resistant states such as obesity and type 2 diabetes, and whether this contributes to insulin resistance in vivo by altering glucose uptake or glycogen synthesis, is unknown. Furthermore, there is no data regarding the ability of insulin to activate Akt 3 in insulin target tissues in humans.

Therefore, to determine the mechanism(s) for insulin resistance in obese nondiabetic and obese type 2 diabetic subjects, we investigated the ability of insulin to stimulate Akt activity in skeletal muscle. Here we report that in vivo infusion of insulin in humans phosphorylates and activates Akt 1 and Akt2 (designated Akt1/2), and tends to activate Akt3, in skeletal muscle. The activity of $\mathrm{Akt} 1 / 2$ and $\mathrm{Akt} 3$ is normal in muscle of insulin-resistant obese subjects and obese type 2 diabetic subjects, despite a 50\% decrease in insulin-stimulated IRS-1-associated PI 3-kinase activity and a 39\% decrease in IRS-2-associated PI 3-kinase activity in obese diabetic subjects. Thus, the key steps involved in insulin resistance in skeletal muscle of moderately obese nondiabetic and type 2 diabetic subjects (i.e., impaired insulin action on glucose uptake and GS activation) do not appear to involve defective stimulation of Akt isoforms.

\section{Methods}

Human subjects. Eight lean nondiabetic subjects, 8 obese nondiabetic subjects, and 12 obese subjects with type 2 diabetes participated in this study. Nearly all subjects were male (Table 1); there were 3 females in the diabetic group. There was no difference in the signaling or insulin-action parameters when the 3 female subjects were excluded from the analyses, so they were included in the analyses. The experimental protocol was approved by the Human Subjects Committee of the University of California-San Diego and of the Veterans Administration Medical Center, San Diego. Written informed consent was obtained from all subjects. All of the nondiabetic subjects had normal glucose tolerance ( $75 \mathrm{~g}$ oral glucose load), as defined by fasting glucose below 126 $\mathrm{mg} / \mathrm{dL}$ and 2-hour glucose below $140 \mathrm{mg} / \mathrm{dL}$ (41). All subjects were screened to ensure that they were healthy (aside from having diabetes) and did not have significant diabetic complications. Hypoglycemic agents were withdrawn at least 2 weeks before studies were performed. No subject was on any other medication that is known to affect carbohydrate metabolism.

Protocol. All study subjects were admitted to the Special Diagnostic and Treatment Unit at the Veterans Administration Medical Center, San Diego. Subjects consumed a standardized weight-maintenance diet containing 55\% of calories as carbohydrate, $30 \%$ as fat, and $15 \%$ as protein, for at least 24 hours before the studies. After an overnight fast, all subjects underwent a 3-hour hyperinsulinemic (120 or $300 \mathrm{mU} / \mathrm{m}^{2}$ per minute), euglycemic (5.0-5.5 mM) clamp as described previously (42). The glucose disposal rate (GDR) was determined during the last 30 minutes of the clamp. Percutaneous needle biopsies of vastus lateralis muscle were performed before insulin infusion and at the end of the clamp study as described previously (42), and muscle tissue was immediately frozen in liquid nitrogen. At each insulin infusion rate, plasma insulin concentrations were similar among all groups. There were no differences in the mean values for insulin-stimulated PI 3-kinase activity, Akt activity, Akt gel mobility shift, GDR, or GS activity between subjects in a given group who were infused at 120 or 300 $\mathrm{mU} / \mathrm{m}^{2}$ per minute. Therefore, data at the 2 infusion rates were combined for each group. In some additional subjects, muscle biopsies were also taken after $15 \mathrm{~min}$ utes of insulin infusion $\left(300 \mathrm{mU} / \mathrm{m}^{2}\right.$ per minute) to determine whether there was an earlier transient peak in PI 3-kinase or Akt activity. Plasma glucose and insulin levels were determined before each biopsy.

PI 3-kinase activity. Fifty milligrams of tissue was homogenized in $500 \mu \mathrm{L}$ lysis buffer, containing $10 \mathrm{mM}$ Tris ( $\mathrm{pH}$ 8.1), $140 \mathrm{mM} \mathrm{NaCl}, 10 \mathrm{mM}$ sodium pyrophosphate, $10 \mathrm{mM} \mathrm{NaF}, 1 \mathrm{mM} \mathrm{CaCl} 2,1 \mathrm{mM} \mathrm{MgCl}_{2}, 2 \mathrm{mM}$ $\mathrm{Na}_{3} \mathrm{VO}_{4}, 10 \%$ glycerol, $1 \% \mathrm{NP}-40,10 \mu \mathrm{g} / \mathrm{mL}$ aprotinin, 50 $\mu \mathrm{M}$ leupeptin, and $2 \mathrm{mM}$ PMSF, at $4^{\circ} \mathrm{C}$ using a Polytron at half maximum speed for 1 minute. It was then solubilized by continuous stirring for 1 hour at $4^{\circ} \mathrm{C}$, and centrifuged for 10 minutes at $14,000 \mathrm{~g}$. The supernatants 
(500 $\mu$ g protein) were immunoprecipitated with $5 \mu \mathrm{L}$ of an IRS- 1 or IRS- 2 antibody (gifts from Morris White, Joslin Diabetes Center, Boston, Massachusetts, USA) coupled to protein A-Sepharose (Sigma Chemical Co., St. Louis, Missouri, USA). The immune complex was washed, and PI 3-kinase activity was measured as described (43) and then quantitated using a PhosphorImager (ImageQuant software; Molecular Dynamics, Sunnyvale, California, USA).

Akt kinase activity. Fifty milligrams of tissue was homogenized on ice in $500 \mu \mathrm{L}$ of buffer A (20 mM Tris [pH 7.5], $5 \mathrm{mM}$ EDTA, $10 \mathrm{mM} \mathrm{Na} \mathrm{P}_{2} \mathrm{O}_{7}, 100 \mathrm{mM} \mathrm{NaF}$, and $2 \mathrm{mM}$ $\mathrm{Na}_{3} \mathrm{VO}_{4}$ ) containing $1 \% \mathrm{NP}-40,1 \mathrm{mM}$ PMSF, and 10 $\mu \mathrm{g} / \mathrm{mL}$ aprotinin, using a Polytron. Muscle lysates were solubilized as described for the PI 3-kinase assay. The supernatants $(500 \mu \mathrm{g}$ protein) were immunoprecipitated for 4 hours at $4^{\circ} \mathrm{C}$ with $4 \mu \mathrm{g}$ of an Akt antibody that recognizes both Akt1 and Akt2 (Upstate Biotechnology Inc., Lake Placid, New York, USA) or with $4 \mu \mathrm{g}$ of an Akt3 antibody (Upstate Biotechnology Inc.) coupled to protein G-Sepharose beads (Amersham Pharmacia Biotech, Piscataway, New Jersey, USA). The immune pellets were washed, and Akt activity was measured as described (44). With this protocol, the efficiency of immunoprecipitation of Akt is approximately 95\% (not shown).

GS activity. Twenty milligrams of muscle biopsy sample was homogenized at $4^{\circ} \mathrm{C}$ with $1 \mathrm{~mL}$ of an extraction buffer (50 mM HEPES, $10 \mathrm{mM}$ EDTA, $100 \mathrm{mM} \mathrm{NaF}, 5$ mM DTT, $1 \mu \mathrm{M}$ leupeptin, $1 \mu \mathrm{M}$ pepstatin, and 200 $\mu \mathrm{M}$ PMSF; $\mathrm{pH} 7.5$ ), using a Polytron at maximum speed for 15 seconds. After centrifugation $(3,000 \mathrm{~g}$ for 20 minutes), the supernatant was diluted with the extraction buffer to a final total protein concentration of $0.5 \mathrm{mg} / \mathrm{mL}$, and GS activity was measured as described $(45,46)$. GS activity was determined at a physiologic concentration of substrate (0.3 mM UDPglucose), calculated as nanomoles of UDP-glucose incorporated into glycogen per minute per milligram of total protein, and expressed as fractional velocity (activity assayed at $0.1 \mathrm{mM}$ glucose-6-phosphate divided by the activity at $10 \mathrm{mM}$ glucose-6-phosphate). This is an indicator of the change in the phosphorylation state of GS in response to insulin (47).

Akt gel mobility shift and $p 85$ protein levels. One hundred micrograms of tissue-lysate protein per lane was resolved by SDS-PAGE ( $8 \%$ gel) and transferred to nitrocellulose membranes (Schleicher \& Schuell Inc., Keene, New Hampshire, USA). The nitrocellulose membranes were blocked with $5 \%$ nonfat dry milk for 1 hour at room temperature, and then incubated with either an Akt antibody that recognizes both Akt 1 and Akt 2 or an antibody against the $\mathrm{p} 85 \alpha$ subunit of PI 3-kinase (both from Upstate Biotechnology Inc.), in $1 \%$ nonfat dry milk overnight at $4^{\circ} \mathrm{C}$. The membranes were washed, and bands were visualized using enhanced chemiluminescence (Amersham Pharmacia Biotech) and quantified by densitometry (Molecular Dynamics).

Statistical analysis. Data calculation and statistical analysis were performed using the StatView program (Abacus Concepts Inc., Berkeley, California, USA). Data are presented as mean \pm SEM. Statistical significance was tested with repeated-measures ANOVA.
Table 1

Clinical and metabolic characteristics of the subjects

$\begin{array}{lccc} & \text { Lean } & \text { Obese } & \text { Diabetic } \\ \text { Age (years) } & 51.7 \pm 5.3 & 44.8 \pm 2.5 & 49.9 \pm 2.6 \\ \text { BMI } & 24.3 \pm 0.6 & 31.7 \pm 1.3^{\mathrm{A}} & 34.0 \pm 1.3^{\mathrm{A}} \\ \text { Hemoglobin A } 1 \mathrm{c}(\%) & 5.2 \pm 0.1 & 4.9 \pm 0.2 & 9.5 \pm 1.1^{\mathrm{B}, \mathrm{C}} \\ \text { Glucose }(\mathrm{mmol} / \mathrm{L}) & 5.1 \pm 0.1 & 5.3 \pm 0.3 & 8.9 \pm 1.0^{\mathrm{B}, \mathrm{D}} \\ \text { Insulin }(\mathrm{pmol} / \mathrm{L}) & 40 \pm 9 & 37 \pm 15 & 117 \pm 20^{\mathrm{B}, \mathrm{D}} \\ \text { FFAs }(\mathrm{mmol} / \mathrm{L}) & 0.51 \pm 0.06 & 0.50 \pm 0.11 & 0.79 \pm 0.11^{\mathrm{E}} \\ \end{array}$

Number of subjects: lean, 8 (all male); obese, 8 (all male); diabetic, 12 (9 male, 3 female). Glucose, insulin, and FFAs were measured after an overnight fast. BMI, body mass index. ${ }^{A} P<0.001$ vs. lean. ${ }^{B} P<0.01$ vs. lean. ${ }^{C} P<0.001$ vs. obese. ${ }^{D} P<$ 0.01 vs. obese. ${ }^{E} P<0.05$ vs. lean.

\section{Results}

Clinical and metabolic characteristics of the subjects. Mean age was similar in the lean, obese nondiabetic, and obese type 2 diabetic subjects (Table 1). Body mass index was significantly increased in obese nondiabetic and obese diabetic subjects compared with lean subjects $(P<$ 0.001). Body mass index was not significantly different between obese nondiabetic and obese diabetic subjects. Hemoglobin $A_{1 c}$, fasting serum glucose, and fasting plasma insulin were significantly elevated in obese diabetic subjects compared with lean or obese nondiabetic subjects. Fasting plasma FFA levels were normal in obese nondiabetic subjects, and were elevated $55-58 \%$ in diabetic subjects, compared with levels in lean and obese nondiabetic subjects $(P<0.05)$. Figure 1 shows that the GDR (as determined by a hyperinsulinemic, euglycemic clamp) was reduced $31 \%$ in the obese nondiabetic subjects compared with lean subjects $(P<0.001) ; 63 \%$ in obese diabetic subjects compared with lean subjects $(P<0.001)$; and $47 \%$ in obese diabetic subjects compared with obese nondiabetic subjects $(P<0.001)$.

Akt kinase activity and phosphorylation, and PI 3-kinase activity. Basal activity of Akt $1 / 2$ was not different among the 3 groups (Figure $2 \mathrm{a}$ ). Insulin increased Akt $1 / 2$ activity in muscle of nearly every subject; the mean increase was 1.7 fold in lean, 2.0-fold in obese nondiabetic, and 1.7-fold in obese diabetic subjects ( $P=$ NS among groups). For 1 lean and 1 obese nondiabetic subject, biopsy specimens were available only in the insulin-stimulated state. Therefore, these 2 values were included without corresponding basal values. These values did not change the means for insulinstimulated Akt1/2 activity, which were similar in all 3

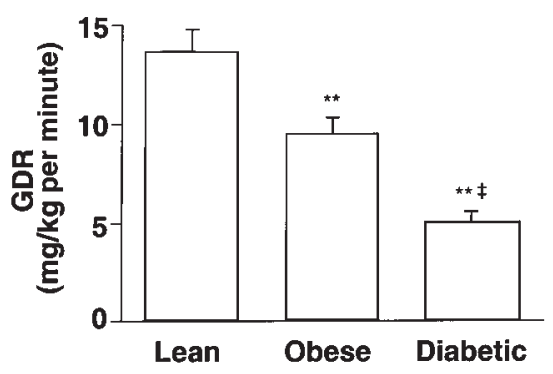

\section{Figure 1}

GDR during hyperinsulinemic, euglycemic clamp in lean, obese nondiabetic, and obese diabetic subjects. Data are mean \pm SEM for $8-12$ subjects per group. ${ }^{*} P<0.001$ vs. lean. $¥ P<0.001$ vs. obese nondiabetic. 


\section{Figure 2}

Akt1/2 kinase activity (a), Akt1/2 phosphorylation (b), Akt $1 / 2$ protein levels (c), and Akt3 kinase activity (d) in skeletal muscle of lean, obese nondiabetic, and obese diabetic subjects. Twenty-six subjects had biopsies of vastus lateralis muscle performed before and at the end of a 3 hour hyperinsulinemic, euglycemic clamp. One additional lean and 1 additional obese subject have data only in the insulin-stimulated state, because biopsies before the clamp gave very low yields. ( $(a$ and $\mathbf{d}$ ) Akt kinase activity was measured in muscle lysates $(500 \mu \mathrm{g})$ that were subjected to immunoprecipitation with either an antibody that recognizes both Akt1 and Akt2, or an Akt3 antibody. The immunoprecipitated pellets were assayed for kinase activity using Crosstide as substrate. In a, each ellipse shows values from a single subject; rectangles show mean \pm SEM for 7-12 subjects per group. In d, bars represent mean \pm SEM for 5 lean, 4 obese, and 8 diabetic subjects. (b and $\mathbf{c}$ ) Proteins in muscle lysates $(100 \mu \mathrm{g})$ were separated by SDS-PAGE on $8 \%$ gels and transferred to nitrocellulose membranes. Akt $1 / 2$ was visualized using immunoblotting. (b) Akt $1 / 2$ gel mobility shift in response to insulin in 3 representative subjects. (c) Representative autoradiogram of Akt $1 / 2$ levels in muscle of 6 subjects; bars show densitometric quantitation of Akt protein levels in 8-12 subjects per group. The autoradiograms in $\mathbf{b}$ and $\mathbf{c}$ are each representative of 3 different gels.

groups. Figure $2 \mathrm{~b}$ shows the gel mobility shift of Akt $1 / 2$ in response to insulin administration in vivo. In muscle from subjects in the basal state, Akt1/2 migrated on SDS-PAGE primarily as 1 band, representing 1 phosphorylation state. In muscle biopsies taken from the same subjects during insulin stimulation, a portion of Akt migrated more slowly because of hyperphosphorylation (48). The gel mobility shift in response to insulin was similar in lean, obese nondiabetic, and obese diabetic subjects. This indicates a normal extent of Akt $1 / 2$ phosphorylation in muscle in response to insulin in insulin-resistant subjects. Figure $2 c$ shows that the total amount of Akt1/2 protein was not significantly altered in skeletal muscle of obese nondiabetic or obese diabetic subjects. The bars show densitometric quantitation for 8-12 subjects per group.

Figure $2 \mathrm{~d}$ shows that Akt3 activity in the basal and insulin-stimulated states was also not different among groups. Insulin tended to increase Akt 3 activity in all groups, but the insulin effect on Akt3, unlike Akt1/2, did not reach statistical significance, owing to greater variability among values in each group (Figure $2 \mathrm{~d}$ ). These data concur with studies in the rat showing minimal stimulation of Akt3 in muscle after insulin injection (30).

To determine whether there was an early transient peak in Akt activity at which time insulin activation might be defective in insulin-resistant subjects, we carried out pilot studies in muscle of a similar group of lean, obese, and obese type 2 diabetic subjects who were biopsied at 15 minutes and again at 3 hours after insulin infusion. Akt $1 / 2$ activity was no higher at 15 minutes than at 3 hours (data not shown), nor was it different among groups at either time point. Maximally stimulating plasma insulin levels $(679-826 \mu \mathrm{U} / \mathrm{mL})$ were achieved in all subjects after 15 minutes of infusion. Therefore, we pursued the studies at 3 hours, as shown above, so that Akt activity was measured at the same time as GDR and GS activity. To determine whether a defect in Akt stimulation might be uncovered at a submaximal insulin concentration, we carried out additional pilot studies, infusing subjects with a dose of a

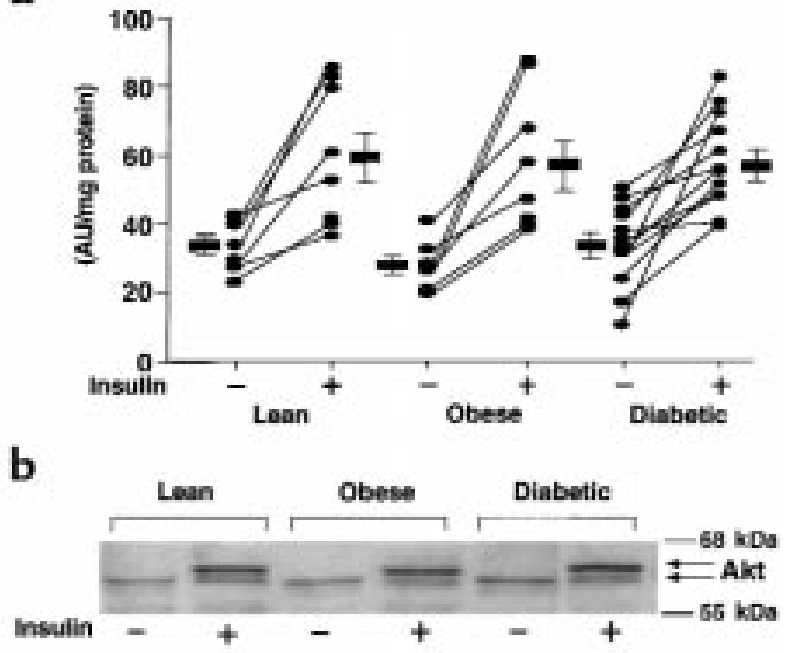

c
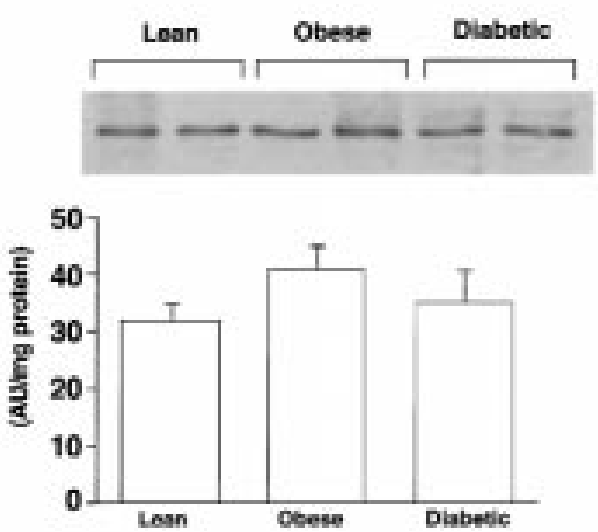

d

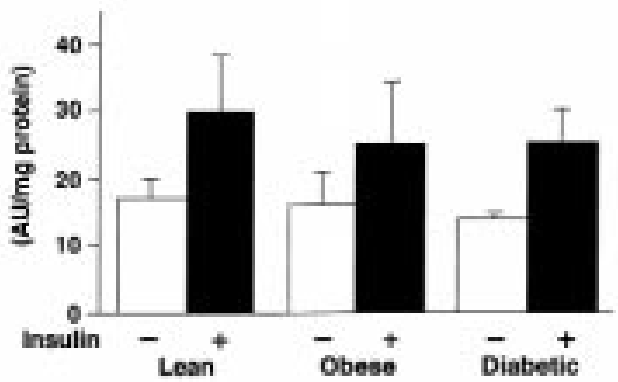

insulin $\left(90 \mathrm{mU} / \mathrm{m}^{2}\right.$ per minute) that gave submaximal stimulation of glucose uptake in obese and diabetic subjects. We found no defect in Akt1/2 stimulation in diabetic subjects at the lower insulin concentration, despite defective stimulation of glucose disposal (data not shown). Although we cannot rule out a subtle shift in the sensitivity of Akt to insulin in insulin-resistant subjects, such a subtle shift would be very difficult to discern. Most importantly, at insulin concentrations in which defective glucose disposal is present in diabetic subjects, there is no defect in Akt $1 / 2$ or Akt 3 activity in muscle.

Figure 3 shows IRS-1- and IRS-2-associated PI 3-kinase activity in muscle from the 3 primary study groups. Figure 3 , a and c show representative autoradiograms in which phosphatidylinositol 3-phosphate is the product of 


\section{Figure 3}

IRS-1-associated (a and $\mathbf{b}$ ) and IRS-2-associated ( $\mathbf{c}$ and $\mathbf{d}$ ) PI 3-kinase activity and p85 protein levels (e) in skeletal muscle of lean, obese nondiabetic, and obese diabetic subjects. All subjects underwent a 3-hour hyperinsulinemic, euglycemic clamp, and biopsies of vastus lateralis muscle were performed before and at the end of the clamp. (a and c) PI 3-kinase activity was measured in muscle lysates $(500 \mu \mathrm{g})$ that were subjected to immunoprecipitation with an IRS-1 (a) or IRS-2 (c) antibody. The origin and position of phosphatidylinositol 3-phosphate (PI3P) are indicated on the left. Each lane contains a sample from a single subject; the basal (-) and insulin-stimulated $(+)$ samples from each subject were loaded in adjacent lanes. The autoradiogram is representative of 3 independent experiments. (b and d) Quantitation of IRS-1-associated (b) or IRS-2-associated (d) PI 3-kinase activity in muscle using a Phosphorlmager. Data are mean \pm SEM for $6-12$ subjects per group. ${ }^{*} P<0.05$ vs. insulin-stimulated obese nondiabetic subjects. $\$ P<0.01$ vs. insulin-stimulated lean subjects. ${ }^{\#} P<0.05$ vs. insulin-stimulated lean subjects. (e) Proteins in muscle lysates $(100 \mu \mathrm{g})$ were separated by SDS-PAGE on $8 \%$ gels and transferred to nitrocellulose membranes. The p 85 regulatory subunit of PI 3-kinase was visualized by immunoblotting with a p $85 \alpha$ antibody, and levels were densitometrically quantitated. The autoradiogram is representative of 3 independent experiments. Each lane contains muscle from 1 subject; bars show mean \pm SEM for 6-12 subjects per group.

the immune complex PI 3-kinase assay. Samples from a single subject biopsied in the basal and insulin-stimulated states were run in adjacent lanes. Figure $3, \mathrm{~b}$ and $\mathrm{d}$ show quantitation of results from many subjects. Basal IRS1-associated PI 3-kinase activity was unchanged among the 3 groups (Figure 3, a and b). Insulin increased IRS1-associated PI 3-kinase activity 6.1-fold in lean, 3.7-fold in obese nondiabetic, and just 2.4-fold in obese diabetic subjects. The reduced fold stimulation in the obese nondiabetic subjects was due primarily to a tendency for increased basal PI 3-kinase activity, but this did not reach statistical significance. Insulin-stimulated IRS-1-associated PI 3-kinase activity was normal in muscle from obese nondiabetic subjects, but was reduced $50 \%$ in obese diabetic subjects compared with lean subjects $(P<0.01)$ and obese nondiabetic subjects $(P<0.05)$. Basal IRS-2-associated PI 3-kinase activity was also not different among groups (Figure 3, c and d). Insulin increased IRS-2-associated PI 3-kinase activity 2.2-fold in lean, 1.6-fold in obese, and 1.4-fold in diabetic subjects $(P<0.05$ vs. lean control; Figure 3d). Insulin-stimulated IRS-2-associated PI 3-kinase activity was reduced $38-39 \%$ in diabetic subjects compared with both lean and obese subjects $(P<0.05)$. The amount of the p85 regulatory subunit of PI 3 -kinase was unchanged in these subjects (Figure $3 e$ ).

GS activity. GS activity has previously been shown to be decreased in muscle of type 2 diabetic subjects (49-51); this is thought to contribute to insulin resistance. In this study, when we found that activation of Akt was normal in diabetic subjects (Figure 2), we investigated whether GS activity was decreased in the same study population. Basal GS activity tended to be reduced $28 \%$ in muscle of nondiabetic obese subjects $(P=\mathrm{NS})$, and was significantly reduced $(49 \%)$ in muscle of obese diabetic subjects $(P<0.05)$ compared with lean subjects (Figure 4$)$. During the clamp, insulin stimulated GS activity by 2 - to 2.5 -fold in all groups. The fractional velocity for insulin-stimulated GS activity was $30 \%$ lower in diabetic subjects than in lean subjects $(P<0.01)$ and obese subjects $(P<0.05)$. a

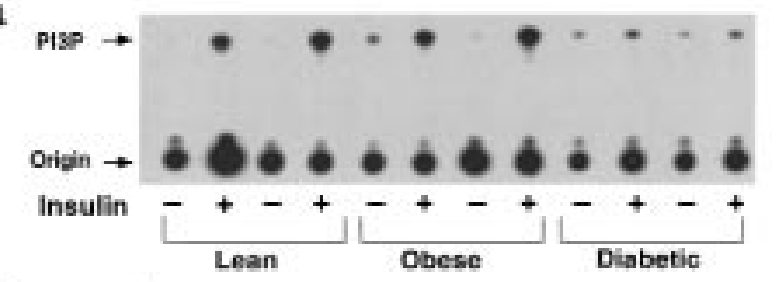

b

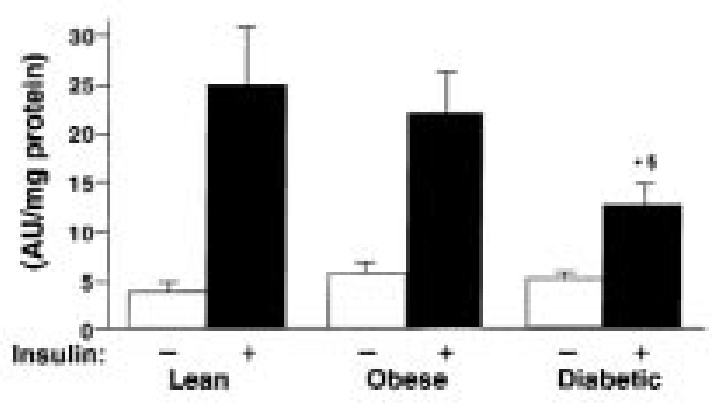

c

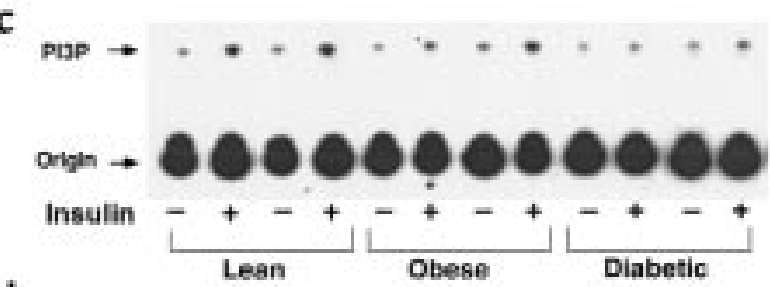

d

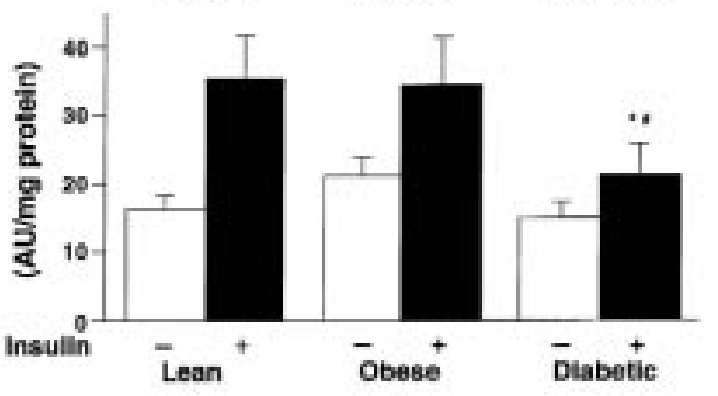

e

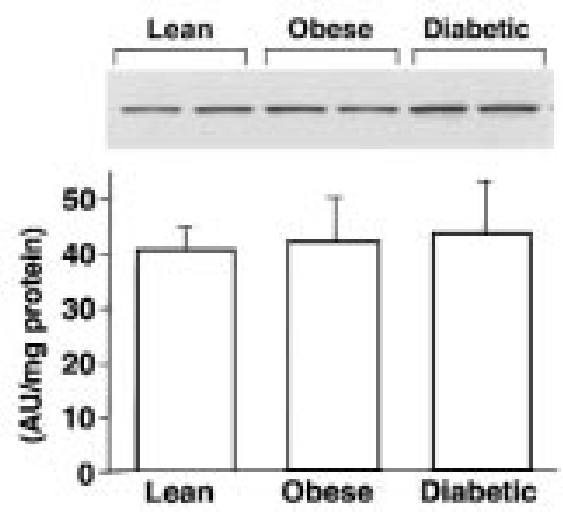

Relationship between Akt activity, PI 3-kinase activity, and $G D R$. A highly significant positive relationship was seen between insulin-stimulated Akt $1 / 2$ activity and insulinstimulated GDR in lean subjects $(r=0.85, P<0.001$; Figure 5a). This correlation was not present in obese nondiabetic or obese diabetic subjects (Figure 5, b and c). Neither the absolute level of insulin-stimulated Akt $1 / 2$ activity nor the increment in insulin-stimulated activity above basal levels correlates with fasting 


\section{Figure 4}

GS activity in skeletal muscle of lean, obese nondiabetic, and obese diabetic subjects. All subjects underwent a 3-hour hyperinsulinemic, euglycemic clamp; biopsies of vastus lateralis muscle were performed before and at the end of the clamp. GS activity was determined at a subsaturating concentration of substrate ( $0.3 \mathrm{mM}$ UDP-glucose) and is expressed as fractional velocity (FV; GS activity at $0.1 \mathrm{mM}$ glucose-6-phosphate divided by the activity at $10 \mathrm{mM}$ glucose-6-phosphate). Data are mean \pm SEM for 6-12 subjects per group. †Basal values in diabetic subjects are different from those in lean subjects $(P<0.05)$. §Insulin-stimulated values in diabetic subjects are different from those in lean subjects $(P<0.01)$. ${ }^{*}$ Insulin-stimulated values in diabetic subjects are different from those in obese nondiabetic subjects $(P<0.05)$.

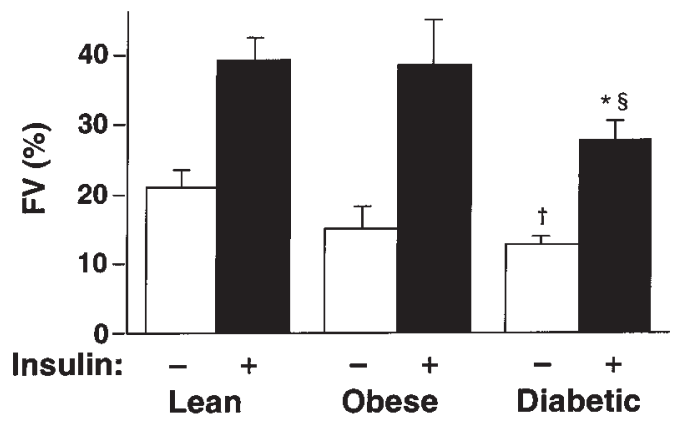

cose disposal and activation of GS in muscle of obese, type 2 diabetic subjects is that Akt may not be the major (or the only) downstream mediator of these metabolic actions of insulin. The role of Akt in insulin-stimulated glucose transport remains controversial, and studies with 2 different dominant-negative constructs of Akt yielded conflicting results $(32,38)$. The demonstration that rapamycin inhibits insulin stimulation of GS (18) suggests that another pathway may be involved in this metabolic action of insulin in addition to, or as an alternative to, Akt-mediated phosphorylation of GSK-3 (34, 53), at least in some cell types.

Only a few studies have addressed the role of Akt in normal insulin action or in insulin-resistant states in intact animals. Glucosamine infusion in rats rapidly impairs insulin-stimulated glucose disposal and PI 3kinase activity in skeletal muscle, but activation of Akt is preserved (54), consistent with our current results in obese diabetic humans. In contrast, in chronically insulin-deficient diabetic rats, Akt activity in muscle (stimulated in vitro by insulin) was diminished $(55,56)$. The effect of insulin on Akt activity was normalized by phlorizin treatment, which improved glycemia and glucose tolerance without altering plasma insulin levels. This suggested that hyperglycemia downregulated Akt activity. The reason for the different results in this model (55) could derive from other metabolic disturbances associated with this chronically insulin-deficient diabetic state, or from differences in study design. Activation of Akt was performed in isolated muscles in vitro, and defects were seen only at supraphysiologic (120 nM) insulin concentrations, not at physiologic concentrations $(0.6$ and $2.4 \mathrm{nM})(40,56)$. In fact, at a physiologic insulin concentration in vitro, PI 3-kinase activity was reduced but Akt activity was normal in soleus muscles of diabetic rats $(40,56)$, consistent with our observation in humans that activation of Akt can be preserved under conditions in which PI 3-kinase activity is impaired.

Also consistent with our current findings are data from a severely insulin-resistant model without hyperglycemia - the Zucker fa/fa obese rat. Insulin-stimulated Akt activity in skeletal muscle of these obese rats was very mildly reduced, whereas PI 3-kinase was reduced 77\% compared with lean rats (Y.-B. Kim et al., manuscript submitted for publication). Taken together, these studies indicate that either (a) only a small amount of PI 3-kinase activity is necessary to maximally activate Akt, or (b) other pathways that are independent of PI 3-kinase are also involved in activation of Akt by insulin, at least in insulin-resistant 
states. Data in support of the first hypothesis come from recent studies in hepatoma cells transduced with a dominant-interfering mutant of dynamin. Insulin-stimulated PI 3-kinase activation was reduced 50-60\% while Akt activation was normal (57). Data relevant to the second hypothesis include the fact that activation of Akt without activation of PI 3-kinase has been shown with growth hormone (25), isoproterenol (26), osmotic shock (27), and changes in intracellular calcium (58). Conceivably, such pathways could be induced in altered metabolic states such as obesity or diabetes.

We considered the possibility that the discrepancy between PI 3-kinase and Akt kinase activities might not be present if we measured a larger pool of insulinresponsive PI 3-kinase activity, such as that associated with phosphotyrosine. However, complete abolition of insulin-stimulated phosphotyrosine-associated PI 3kinase activity has been demonstrated in muscle of type 2 diabetic humans (9). Furthermore, we (54) and others $(59,60)$ have found parallel or even greater reductions in phosphotyrosine-associated PI 3-kinase activity compared with IRS-1-associated PI 3-kinase activity in skeletal muscle of insulin-resistant rodents. In this study we also found a defect in IRS-2-associated PI 3-kinase activity in muscle of diabetic subjects. Interestingly, the stimulatory effect of insulin on IRS-2-associated PI 3-kinase activity in all groups, including lean controls, was lower than the effect on IRS-1-associated PI 3-kinase activity. This is consistent with the findings in IRS-1 and IRS-2 knockout mice, suggesting a greater role for IRS- 1 in insulin-stimulated glucose uptake in muscle $(61,62)$.

Other possible mechanisms for discordance between PI 3-kinase and Akt could involve regulation distal to activation of PI 3-kinase. For example, increased activity of phosphoinositide-dependent protein kinases that activate Akt (28), or decreased activity of phosphatases that dephosphorylate phospholipid products of PI 3kinase (63), could amplify the signal from PI 3-kinase. Whether such alterations are present in muscle of diabetic subjects, and whether they could result in preserved activation of Akt when PI 3-kinase activity is impaired, are important questions for future studies.

The activity of PI 3-kinase (Y.-B. Kim and B.B. Kahn, unpublished observations) and Akt $(30,53)$ after insulin bolus injection in rats can rapidly peak and fall to a lower plateau, possibly because of the rapid clearance of insulin. However, because transient stimulation of PI 3- kinase activity has also been shown in rat soleus muscle in vitro, even with sustained exposure to high levels of insulin (64), we considered whether stimulation of these signaling pathways could be transient during the insulin clamp. The timing of PI 3-kinase activation has been studied in normal, lean humans under insulin-clamp conditions (65). Stimulation of PI 3-kinase activity after 15 minutes of insulin infusion tended to be lower than after 1 hour, despite the fact that comparably high plasma insulin levels were achieved after 15 minutes (65). The magnitude of antiphosphotyrosine-associated PI 3kinase stimulation after 40 minutes (9) or 1 hour of insulin clamp (65) was similar to the stimulation of IRS1-associated PI 3-kinase activity that we saw after 3 hours in lean subjects (Figure 3 ).

The timing of Akt activation by insulin has not been reported in humans. Therefore, we carried out the pilot studies described in the Results section above. Because we did not find higher stimulation of Akt kinase activity after 15 minutes than after 3 hours, we pursued studies after full steady-state conditions, which allowed us to directly compare effects on insulin signaling with concurrent metabolic responses. In additional pilot studies with lower insulin infusion rates, which gave submaximal stimulation of glucose disposal in diabetic subjects, we also found normal stimulation of Akt activity. Although a subtle shift in the dose response of Akt activation by insulin in diabetic subjects cannot be excluded, our data show that at insulin concentrations that are associated with marked resistance to the stimulation of glucose disposal and GS in diabetic subjects, Akt activation and phosphorylation are normal.

Investigation of steps downstream of Akt could shed light on the role of Akt in insulin resistance. GSK-3 can be phosphorylated and inactivated by Akt; its inactivation results in stimulation of GS. Recent data in a cohort of subjects similar to those studied here indicate that the protein levels and total activity of GSK- $3 \alpha$ and GSK-3 $\beta$ are increased in muscle of diabetic subjects in both the basal and insulin-stimulated states (S.E. Nikoulina et al., manuscript submitted for publication). However, the specific activity of GSK-3 (activity per molecule of enzyme) is normal in diabetic subjects, and GSK- $3 \alpha$ activity falls normally in response to insulin, consistent with our demonstration here that basal and insulinstimulated Akt activity is normal in muscle of diabetic subjects. Furthermore, the total activity of GSK-3 corre-
Figure 5

Relationship between insulin-stimulated Akt1/2 kinase activity in skeletal muscle and GDR in vivo in lean, obese nondiabetic, and obese diabetic subjects. Each square represents data from 1 subject.
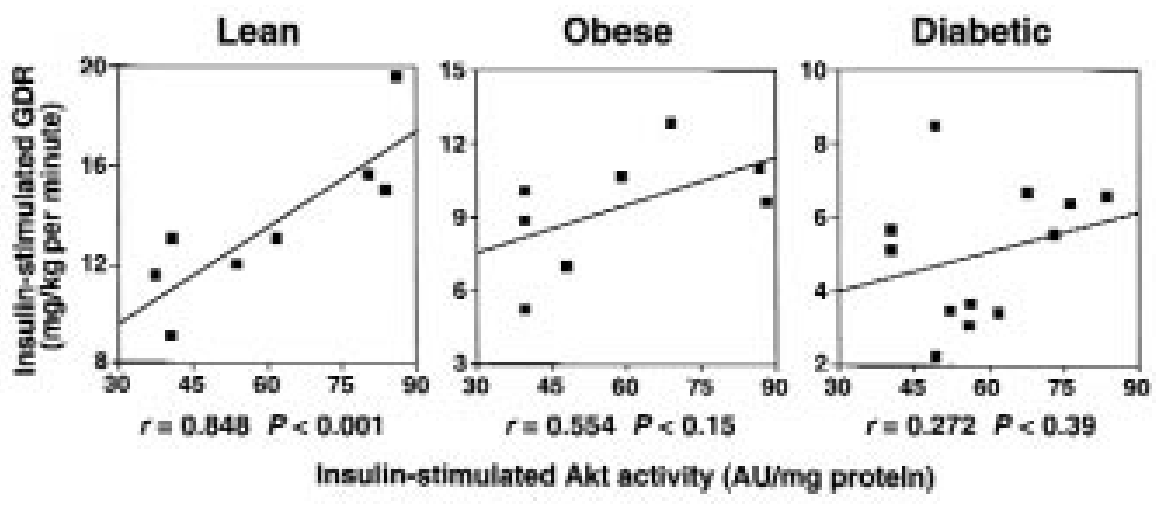
lates inversely with both GS activity and insulin-stimulated glucose disposal (S.E. Nikoulina et al., manuscript submitted for publication). Thus, the reduced GS activity in muscle of diabetic subjects may be explained by increased GSK-3 expression, rather than a defect in the ability of insulin to activate Akt.

In our obese subjects, we did not see a defect in insulin activation of PI 3-kinase or GS, although such defects have been reported in more severely insulin-resistant obese subjects $(43,51)$. We cannot rule out the possibility that obese nondiabetic subjects with more severe insulin resistance would also have impaired PI 3-kinase activity.

In summary, in vivo administration of insulin in humans stimulates Akt $1 / 2$ activity in skeletal muscle and tends to stimulate Akt 3 activity. In insulin-resistant obese subjects with type 2 diabetes, and with the characteristic impairments in glucose disposal and GS activity in skeletal muscle, insulin-stimulated phosphorylation and activation of Akt isoforms are normal. This occurs despite a $50 \%$ reduction in insulin-stimulated IRS1 -associated PI 3-kinase activity and a 39\% reduction in IRS-2-associated PI 3-kinase activity. This suggests that either a PI 3-kinase-independent pathway is involved in stimulation of Akt by insulin in vivo, or that other factors can modulate the effect of PI 3-kinase on Akt. Importantly, whereas defective stimulation of PI 3kinase may play a role in the pathogenesis of insulin resistance, Akt is unlikely to be a major factor in the resistance to insulin action on glucose disposal or GS activation in muscle of obese type 2 diabetic subjects.

\section{Acknowledgments}

This work was supported by National Institute of Diabetes and Digestive and Kidney Diseases grant NIH DK43051; a grant from the American Diabetes Association; funds from the Medical Research Service, Department of Veterans Affairs and Veterans Affairs San Diego Health Care System; and grant MO1 RR-00827 from the General Clinical Research Branch, Division of Research Resources, National Institutes of Health. Y.-B. Kim was supported by a Uehara Memorial Foundation Research Fellowship and by the American Diabetes Association. S.E. Nikoulina was supported by a Career Development Award from the American Diabetes Association. We thank Morris White for the IRS-1 and IRS-2 antibodies.

1. DeFronzo, R.A., Bonadonna, R.C., and Ferrannini, E. 1992. Pathogenesis of NIDDM. A balanced overview. Diabetes Care. 15:318-368.

2. Kahn, B.B. 1998. Type 2 diabetes: when insulin secretion fails to compensate for insulin resistance. Cell. 92:593-596.

3. Yki-Jarvinen, H., Sahlin, K., Ren, J.M., and Koivisto, V.A. 1990. Localization of rate-limiting defect for glucose disposal in skeletal muscle of insulinresistant type I diabetic patients. Diabetes. 39:157-167.

4. Heesom, K.J., Harbeck, M., Kahn, C.R., and Denton, R.M. 1997. Insulin action on metabolism. Diabetologia. 40:B3-B9.

5. White, M.F. 1997. The insulin signalling system and the IRS proteins. Diabetologia. 40:S2-S17.

6. Zierath, J.R., et al. 1996. Insulin action on glucose transport and plasma membrane GLUT4 content in skeletal muscle from patients with NIDDM. Diabetologia. 39:1180-1189.

7. Zierath, J.R, Houseknecht, K.L., and Kahn, B.B. 1996. Glucose transporters and diabetes. Semin. Cell Dev. Biol. 7:295-307.

8. Carey, J.O., Azevedo, J., Jr., Morris, P.G., Pories, W.J., and Dohm, G.L. 1995. Okadaic acid, vanadate, and phenylarsine oxide stimulate 2-deoxyglucose transport in insulin-resistant human skeletal muscle. Diabetes. 44:682-688.

9. Bjornholm, M., Kawano, Y., Lehtihet, M., and Zierath, J.R. 1997. Insulin receptor substrate-1 phosphorylation and phosphatidylinositol 3-kinase activity in skeletal muscle from NIDDM subjects after in vivo insulin stimulation. Diabetes. 46:524-527.

10. Myers, M.G.,Jr., and White, M.F. 1996. Insulin signal transduction and the IRS proteins. Annu. Rev. Pharmacol. Toxicol. 36:615-658.

11. Holman, G.D., and Kasuga, M. 1997. From receptor to transporter: insulin signalling to glucose transport. Diabetologia. 40:991-1003.

12. Lavan, B.E., et al. 1997. A novel $160-\mathrm{kDa}$ phosphotyrosine protein in insulin-treated embryonic kidney cells is a new member of the insulin receptor substrate family. J. Biol. Chem. 272:21403-21407.

13. Lavan, B.E., Lane, W.S., and Lienhard, G.E. 1997. The 60-kDa phosphotyrosine protein in insulin-treated adipocytes is a new member of the insulin receptor substrate family. J. Biol. Chem. 272:11439-11443.

14. Cheatham, B., et al. 1994. Phosphatidylinositol 3-kinase activation is required for insulin stimulation of pp70 S6 kinase, DNA synthesis, and glucose transporter translocation. Mol. Cell. Biol. 14:4902-4911.

15. Okada, T., Kawano, Y., Sakakibara, T., Hazeki, O., and Ui, M. 1994. Essential role of phosphatidylinositol 3-kinase in insulin-induced glucose transport and antilipolysis in rat adipocytes. Studies with a selective inhibitor wortmannin. J. Biol. Chem. 269:3568-3573.

16. Le Marchand-Brustel, Y., Gautier, N., Cormont, M., and Van Obberghen, E. 1995. Wortmannin inhibits the action of insulin but not that of okadaic acid in skeletal muscle: comparison with fat cells. Endocrinology. 136:3564-3570.

17. Hara, K., et al. 1994. 1-phosphatidylinositol 3-kinase activity is required for insulin-stimulated glucose transport but not for RAS activation in $\mathrm{CHO}$ cells. Proc. Natl. Acad. Sci. USA. 91:7415-7419.

18. Shepherd, P.R., Nave, B.T., and Siddle, K. 1995. Insulin stimulation of glycogen synthesis and glycogen synthase activity is blocked by wortmannin and rapamycin in 3T3-L1 adipocytes: evidence for the involvement of phosphoinositide 3-kinase and p70 ribosomal protein-S6 kinase. Biochem. J. 305:25-28.

19. Mendez, R., Myers, M., Jr., White, M.F., and Rhoads, R.E. 1996. Stimulation of protein synthesis, eukaryotic translation initiation factor $4 \mathrm{E}$ phosphorylation, and PHAS-I phosphorylation by insulin requires insulin receptor substrate 1 and phosphatidylinositol 3-kinase. Mol. Cell. Biol. 16:2857-2864.

20. Sutherland, C., Waltner-Law, M., Gnudi, L., Kahn, B.B., and Granner, D.K. 1998. Activation of the ras mitogen-activated protein kinase-ribosomal protein kinase pathway is not required for the repression of phosphoenolpyruvate carboxykinase gene transcription by insulin. J. Biol. Chem. 273:3198-3204.

21. Frevert, E.U., and Kahn, B.B. 1997. Differential effects of constitutively active phosphatidylinositol 3- kinase on glucose transport, glycogen synthase activity, and DNA synthesis in 3T3-L1 adipocytes. Mol. Cell. Biol. 17:190-198.

22. Tanti, J.F., et al. 1996. Overexpression of a constitutively active form of phosphatidylinositol 3-kinase is sufficient to promote Glut 4 translocation in adipocytes. J. Biol. Chem. 271:25227-25232.

23. Bellacosa, A., Testa, J.R., Staal, S.P., and Tsichlis, P.N. 1991. A retroviral oncogene, akt, encoding a serine-threonine kinase containing an SH2-like region. Science. 254:274-277.

24. Franke, T.F., Tartof, K.D., and Tsichlis, P.N. 1994. The SH2-like Akt homology $(\mathrm{AH})$ domain of $\mathrm{c}$-akt is present in multiple copies in the genome of vertebrate and invertebrate eukaryotes. Cloning and characterization of the Drosophila melanogaster c-akt homolog Dakt1. Oncogene. 9:141-148.

25. Sakaue, H., et al. 1997. Phosphoinositide 3-kinase is required for insulininduced but not for growth hormone- or hyperosmolarity-induced glucose uptake in 3T3-L1 adipocytes. Mol. Endocrinol. 11:1552-1562.

26. Moule, S.K., et al. 1997. Regulation of protein kinase B and glycogen synthase kinase-3 by insulin and beta-adrenergic agonists in rat epididymal fat cells. Activation of protein kinase B by wortmannin-sensitive and -insensitive mechanisms. J. Biol. Chem. 272:7713-7719.

27. Konishi, H., et al. 1996. Activation of RAC-protein kinase by heat shock and hyperosmolarity stress through a pathway independent of phosphatidylinositol 3-kinase. Proc. Natl. Acad. Sci. USA. 93:7639-7643.

28. Alessi, D.R., and Cohen, P. 1998. Mechanism of activation and function of protein kinase B. Curr. Opin. Genet. Dev. 8:55-62.

29. Alessi, D.R., et al. 1997. Characterization of a 3-phosphoinositide-dependent protein kinase which phosphorylates and activates protein kinase $\mathrm{B}$ alpha. Curr. Biol. 7:261-269.

30. Walker, K.S., et al. 1998. Activation of protein kinase B beta and gamma isoforms by insulin in vivo and by 3-phosphoinositide-dependent protein kinase-1 in vitro: comparison with protein kinase B alpha. Biochem. J. 331:299-308.

31. Franke, T.F., Kaplan, D.R., and Cantley, L.C. 1997. PI3K: downstream AKT ion blocks apoptosis. Cell. $\mathbf{8 8}: 435-437$.

32. Kitamura, T., et al. 1998. Requirement for activation of the serine-threonine kinase akt (protein kinase B) in insulin stimulation of protein synthesis but not of glucose transport. Mol. Cell. Biol. 18:3708-3717.

33. Hajduch, E., Alessi, D.R., Hemmings, B.A., and Hundal, H.S. 1998. Constitutive activation of protein kinase $\mathrm{B}$ alpha by membrane targeting promotes glucose and system $A$ amino acid transport, protein synthesis, and inactivation of glycogen synthase kinase 3 in L6 muscle cells. Diabetes. 
47:1006-1013.

34. Cross, D.A., Alessi, D.R., Cohen, P., Andjelkovich, M., and Hemmings, B.A. 1995. Inhibition of glycogen synthase kinase-3 by insulin mediated by protein kinase B. Nature. 378:785-789.

35. Cohen, P. 1985. The role of protein phosphorylation in the hormonal control of enzyme activity. Eur. J. Biochem. 151:439-448.

36. Kohn, A.D., Takeuchi, F., and Roth, R.A. 1996. Akt, a pleckstrin homology domain containing kinase, is activated primarily by phosphorylation. J. Biol. Chem. 271:21920-21926.

37. Cong, L., et al. 1997. Physiological role of Akt in insulin-stimulated translocation of GLUT4 in transfected rat adipose cells. Mol. Endocrinol. 11:1881-1890.

38. Wang, Q., et al. 1999. Protein kinase B/Akt participates in GLUT4 translocation by insulin in L6 myoblasts. Mol. Cell. Biol. 19:4008-4018.

39. Ueki, K., et al. 1998. Potential role of protein kinase B in insulin-induced glucose transport, glycogen synthesis, and protein synthesis. J. Biol. Chem. 273:5315-5322.

40. Krook, A., Roth, R.A.,Jiang, X.J., Zierath, J.R., and Wallberg-Henriksson, H. 1998. Insulin-stimulated Akt kinase activity is reduced in skeletal muscle from NIDDM subjects. Diabetes. 47:1281-1286.

41. 1998. Report of the expert committee on the diagnosis and classification of diabetes mellitus. Diabetes Care. 21(Suppl. 1):S5-S22.

42. Thorburn, A.W., Gumbiner, B., Bulacan, F., Brechtel, G., and Henry, R.R. 1991. Multiple defects in muscle glycogen synthase activity contribute to reduced glycogen synthesis in non-insulin dependent diabetes mellitus. $J$. Clin. Invest. 87:489-495.

43. Goodyear, L.J., et al. 1995. Insulin receptor phosphorylation, insulin receptor substrate-1 phosphorylation, and phosphatidylinositol 3-kinase activity are decreased in intact skeletal muscle strips from obese subjects. J. Clin. Invest. 95:2195-2204.

44. Tanti, J.F., et al. 1997. Potential role of protein kinase B in glucose transporter 4 translocation in adipocytes. Endocrinology. 138:2005-2010.

45. Thorburn, A.W., Gumbiner, B., Bulacan, F., Wallace, P., and Henry, R.R. 1990. Intracellular glucose oxidation and glycogen synthase activity are reduced in non-insulin-dependent (type II) diabetes independent of impaired glucose uptake. J. Clin. Invest. 85:522-529.

46. Henry, R.R., Abrams, L., Nikoulina, S., and Ciaraldi, T.P. 1995. Insulin action and glucose metabolism in nondiabetic control and NIDDM subjects. Comparison using human skeletal muscle cell cultures. Diabetes. 44:936-946.

47. Roach, R.J., and Larner, J. 1977. Covalent phosphorylation in the regulation glycogen synthase activity. Mol. Cell. Biochem. 15:179-200.

48. Kohn, A.D., Kovacina, K.S., and Roth, R.A. 1995. Insulin stimulates the kinase activity of RAC-PK, a pleckstrin homology domain containing ser/thr kinase. EMBOJ. 14:4288-4295.

49. Mandarino, L.J., Consoli, A., Jain, A., and Kelley, D.E. 1996. Interaction of carbohydrate and fat fuels in human skeletal muscle: impact of obesity and
NIDDM. Am. J. Physiol. 270:E463-E470.

50. Johnson, A.B., et al. 1991. Impaired activation of skeletal muscle glycogen synthase in non-insulin-dependent diabetes mellitus is unrelated to the degree of obesity. Metabolism. 40:252-260.

51. Damsbo, P., Vaag, A., Hother-Nielsen, O., and Beck-Nielsen, H. 1991. Reduced glycogen synthase activity in skeletal muscle from obese patients with and without type 2 (non-insulin-dependent) diabetes mellitus. Diabetologia. 34:239-245.

52. Cheatham, B., and Kahn, C.R. 1995. Insulin action and the insulin signaling network. Endocr. Rev. 16:117-142.

53. Cross, D.A., et al. 1997. Insulin activates protein kinase B, inhibits glycogen synthase kinase-3 and activates glycogen synthase by rapamycininsensitive pathways in skeletal muscle and adipose tissue. FEBS Lett. 406:211-215.

54. Kim, Y.-B., et al. 1999. Glucosamine infusion in rats rapidly impairs insulin stimulation of phosphoinositol 3-kinase but does not alter activation of Akt/protein kinase B in skeletal muscle. Diabetes. 48:310-320.

55. Krook, A., et al. 1997. Improved glucose tolerance restores insulin-stimulated Akt kinase activity and glucose transport in skeletal muscle from diabetic Goto-Kakizaki rats. Diabetes. 46:2110-2114.

56. Song, X.M., et al. 1999. Muscle fiber type-specific defects in insulin signal transduction to glucose transport in diabetic GK rats. Diabetes. 48:664-670.

57. Ceresa, B.P., Kao, A.W., Santeler, S.R., and Pessin, J.E. 1998. Inhibition of clathrin-mediated endocytosis selectively attenuates specific insulin receptor signal transduction pathways. Mol. Cell. Biol. 18:3862-3870.

58. Yano, S., Tokumistu, H., and Soderling, T.R. 1998. Calcium promotes cell survival through CaM-K kinase activation of the protein-kinase-B pathway. Nature. 396:584-587.

59. Kerouz, N.J., Horsch, D., Pons, S., and Kahn, C.R. 1997. Differential regulation of insulin receptor substrates-1 and -2 (IRS-1 and IRS-2) and phosphatidylinositol 3-kinase isoforms in liver and muscle of the obese diabetic (ob/ob) mouse. J. Clin. Invest. 100:3164-3172.

60. Jullien, D., Heydrick, S.J., Gautier, N., Van Obberghen, E., and Le MarchandBrustel, Y. 1996. Effect of IGF-1 on phosphatidylinositol 3-kinase in soleus muscle of lean and insulin-resistant obese mice. Diabetes. 45:869-875.

61. Withers, D.J., et al. 1998. Disruption of IRS-2 causes type 2 diabetes in mice. Nature. 391:900-904.

62. Previs, S.F., Ren, J.-M., White, M.F., and Shulman, G.I. 1999. Contrasting effects of IRS1 vs. IRS2 gene disruption on carbohydrate and lipid metabolism in vitro. Diabetes. 48(Suppl. 1):A51. (Abstr.)

63. Stambolic, V., et al. 1998. Negative regulation of PKB/Akt-dependent cell survival by the tumor suppressor PTEN. Cell. 95:29-39.

64. Heydrick, S.J., et al. 1993. Defect in skeletal muscle phosphatidylinositol3-kinase in obese insulin-resistant mice. J. Clin. Invest. 91:1358-1366.

65. Hickey, M.S., et al. 1997. Insulin activation of phosphatidylinositol 3-kinase in human skeletal muscle in vivo. J. Appl. Physiol. 83:718-722. 